Ann. Biol. anim. Bioch. Biophys., 1979, 19 (3 B), 813-817.

\title{
Variations de l'activité $\alpha$-amylasique pariétale et intraluminale dans le tube digestif de lapins témoins et à canal pancréatique ligaturé
}

\author{
par Jacqueline CATALA, R. BONNAFOUS
}

Instifut de Physiologie, 2, rue François-Magendie

31400 Toulouse.

Summary. $\alpha$-amylase activity variations in the parietal and intraluminal contents of the digestive tract in control and pancreatic duct ligated rabbits.

$\alpha$-amylase activity (E.C.3 2.1.1) was studied in the parietal and intraluminal contents of different parts of the digestive tract in control $(T)$ and pancreatic duct ligated rabbits $\left(\mathrm{P}^{-}\right)$. Maximal activity at the parietal level in control rabbits was observed in the median part of the duodenum ( $392 \pm 37 \mu \mathrm{M} / \mathrm{maltose} / \mathrm{g} / \mathrm{min}$ at $\left.37^{\circ} \mathrm{C}\right)$. Weak activity ( $3 \mathrm{p} .100$ of the duodenal activity) was seen in other parts of the small and large bowel. Variations in $\alpha$-amylase activity were noted in the post-ligature period in rabbits. This activity declined by $38 \mathrm{p} .100$ 2 weeks after ligature; normal values reappeared 6 weeks later, and 10 weeks after the operation the values were 40 p. 100 higher than those of control rabbits. Maximal intraluminal content activity was observed in the jejunum and ileum of T rabbits $(182+26$ and $92 \pm 6 \mu \mathrm{M} / \mathrm{maltose} / \mathrm{g} / \mathrm{min}$ at $37^{\circ} \mathrm{C}$ ). The large bowel showed only $5 \mathrm{p} .100$ of the jejunal activity. In $P$ - rabbits, $\alpha$-amylase activity progressively increased only in the large bowel from 2 and 6 weeks $(\times 3)$ to 10 weeks $(\times 6)$. Suppression of pancreatic digestion was followed by a compensative process. Hyperplasia and hypertrophy of Brünner's glands could explain the rise in $\alpha$-amylase activity at the duodenal level. The elevation observed in intraluminal contents was certainly of bacterial origin.

Il est connu que chez des animaux qui présentent des troubles pancréatiques, il apparaît souvent une variation des activités enzymatiques intestinales. Plus précisément, dans le cas de déficience pancréatique exocrine, certains auteurs ont pu constater une élévation des hydrolases glucidiques, principalement des disaccharidases (Cerda, Preiser ef Crane, 1972 ; Madzarovova-Nohejlova, 1976 chez l'homme; Alpers ef Tedesco, 1975, chez le rat). Arvanitakis et Olsen (1973) chez l'homme ef le lapin ont également étudié l'évolution des disaccharidases, mais après ligature du canal pancréatique.

II semble cependant que l'activité $\alpha$-amylasique ait suscité beaucoup moins d'intérêt, bien qu'une telle activité intestinale ait été reconnue chez un bon nombre d'espèces (McMichael et Dahlquist, 1968, chez l'homme ; Alpers ef Solin, 1970 chez le rat ; Roberts, 1974, chez le cheval). 
Le but de ce travail est donc de rechercher s'il existe des processus de compensation enzymatique chez le lapin privé de digestion pancréatique. De l'étude enzymologique effectuée chez des lapins à canal pancréatique ligaturé $\left(\mathrm{P}^{-}\right)$nous ne rapportons que les résultats concernant l'activité $\alpha$-amylasique totale pariétale et intraluminale. Nous avons suivi cette activité à la fois chez les animaux témoins (T) et $\mathrm{P}$-, à différentes périodes post-ligature 2,6 et 10 semaines.

\section{Matériel et méthode.}

Cette expérience a été conduite sur 36 lapins mâles $(2,5 \mathrm{~kg}$ au départ de l'expérimentation) : $18 \mathrm{~T}$ et $18 \mathrm{P}$-. Les animaux sont sacrifiés à $9 \mathrm{~h}$, sans être soumis à un jeûne préalable par groupes de $6 \mathrm{~T}$ et $6 \mathrm{P}^{-}$, aux périodes 2,6 et 10 semaines postligature.

Les échantillons pariétaux sont prélevés à 8 niveaux du tube digestif : duodénum $\left(D_{1} D_{2} D_{3}\right)$, jéjunum $(J)$, iléon $(l)$, cæcum $(C)$, côlon proximal $\left(C_{1}\right)$ et côlon distal $\left(C_{2}\right)$.

Les échantillons du matériel intraluminal sont recueillis dans les 6 segments et réservoirs : duodénum (D), jéjunum $(J)$, iléon $(I)$, cæcum $(C)$, côlon proximal $\left(C_{1}\right)$, côlon distal $\left(\mathrm{C}_{2}\right)$.

L'activité $\alpha$-amylasique est estimée selon la méthode de Bernfeld (1951). Les résultats sont exprimés en $\mu$ Moles de maltose libéré $/ \mathrm{g} / \mathrm{MF} / \mathrm{mn}$.

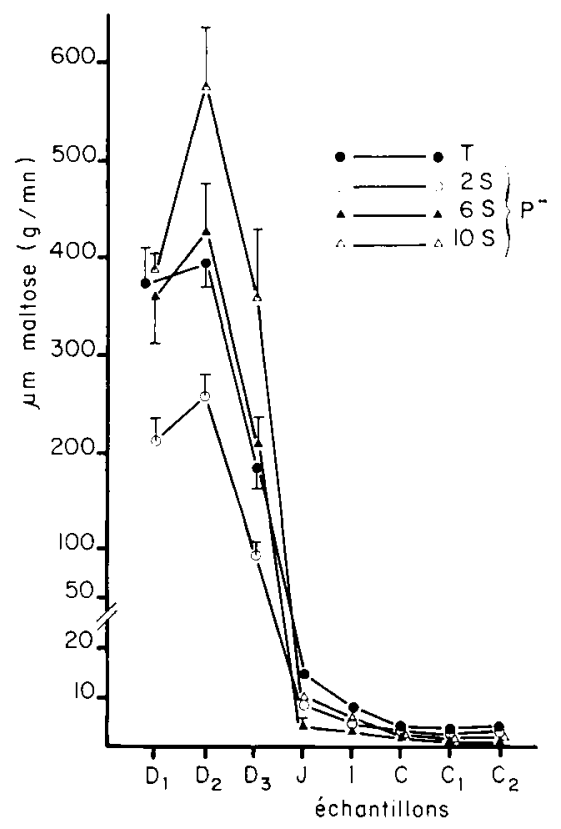

FIG. 1. - Activité $\alpha$-amylasique totale pariétale chez les animaux $T$ ef $P-2,6$ et 10 semaines post-ligature le long du fube digestif : duodénum $\left(D_{1} D_{2} D_{3}\right)$, jéjunum (J), iléon (l), caecum (C), côlon proximal $\left(C_{1}\right)$, côlon distal $\left(C_{2}\right)$. 


\section{Résultats et discussion.}

Activité amylasique pariétale.

De la figure 1 il ressort que la plus forte activité amylasique, chez le lapin témoin, se situe dans le duodénum, plus précisément dans la partie médiane. Dans la partie distale, le taux diminue de moitié, par la suite les activités s'effondrent pour ne plus représenter que 4 p. 100 dans le jéjunum, 2 p. 100 dans l'iléon et 1 p. 100 dans le gros intestin (par rapport à la plus forte activité duodénale).

Plusieurs auteurs signalent avoir une certaine difficulté pour l'estimation réelle de cette activité amylasique intestinale du fait d'une contamination par l'amylase pancréatique (Alpers et Solin, 1970 ; Roberts, 1974), ce qui ne semble pas être le cas chez le lapin. En effet, l'absence d'activité amylasique pariétale au niveau du jéjunum, malgré une forte teneur du matériel intraluminal à ce même niveau, nous permet de penser que le processus d'adsorption de l'amylase pancréatique ne se manifeste pas chez cet animal.

De plus, l'existence éventuelle d'un reflux ne peut être envisagée : tout d'abord, parce que le simple flux intraluminal, comme nous venons de le mentionner, n'entraîne pas de contamination pariétale, et en outre, nous n'avons jamais observé d'activité $\alpha$-chymotrypsique d'origine spécifiquement pancréatique dans le matériel situé en amont de l'abouchement du canal pancréatique.

Donc, chez le lapin, le fait d'observer une activité $\alpha$-amylasique en amont de l'abouchement du canal de Wirsung, nous permet de penser qu'il s'agit bien d'une amylase intestinale différente de l'amylase pancréatique.

Chez les animaux $\mathrm{P}^{-}$, la ligature du canal de Wirsung entraîne des variations de l'activité amylasique duodénale différente selon la période post-ligature considérée.

Dès la $2^{e}$ semaine, nous observons une diminution significative de 38 p. 100 de cette activité (activité globale duodénale $191 \pm 25$ pour les $\mathrm{P}$ - contre $313 \pm 37 \mathrm{U} / \mathrm{g} / \mathrm{pa}$ roi pour les $T$ ). A la 6 e semaine, nous retrouvons des valeurs sensiblement de l'ordre de celles des $T$. A la $10^{\mathrm{e}}$ semaine, nous relevons une augmentation, toujours par rapport aux $T$ significative de 41 p. 100.

Ces résultats sont intéressants à retenir car ils permettent de montrer que, contrairement à ce qui a été décrit pour d'autres enzymes (Alpers ef Tedesco, 1975), cette augmentation d'activițé amylasique ne peut être attribuée à des processus cumulatifs au niveau de la bordure en brosse en absence d'effet désorbant du suc pancréatique. Mais, par contre, à la suite d'une étude histologique effectuée parallèlement à ceffe étude enzymologique, nous avons pu constater qu'il apparaît, corrélativement à cette augmentation d'activité amylasique, une hypertrophie et hyperplasie de la glande de Brünner à ce même niveau duodénal (résultats non publiés).

\section{Activité amylasique intraluminale.}

Chez les lapins $T$, c'est essentiellement au niveau jéjunal que nous obtenons la plus forte activité amylasique du digesta. Cette activité chute très rapidement dans I'iléon ( 50 p. 100), dès le cæcum, elle ne représente plus que 8 p. 100 et 3 p. 100 dans le côlon. Cette activité dans le jéjunum est due à l'a-amylase pancréatique qui s'écoule à 
ce niveau. Les valeurs très faibles relevées dans le segment lui faisant suite, sembleraient montrer que l'activité $\alpha$-amylasique bactérienne est relativement faible chez le lapin normal.

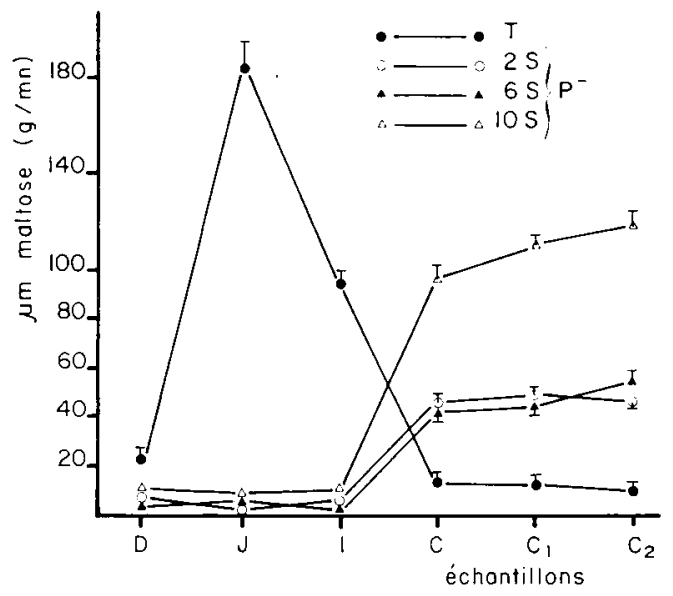

FIG. 2. - Activité $\alpha$-amylasique intraluminale totale chez les animaux $T$ et $P-2,6$ et 10 semaines postligature le long du fube digestif : duodénum (D), jéjunum (J), iléon (I), caecum (C), côlon proximal $\left(C_{1}\right)$, côlon distal $\left(C_{2}\right)$.

L'absence de suc pancréatique entraîne, cette fois, une élévation de l'activité amylasique dès la $2^{\mathrm{e}}$ semaine post-ligature au niveau du gros intestin. Cette augmentation est très importante $\left(\times 3\right.$ à la $2^{\mathrm{e}}$ et $6^{\mathrm{e}}$ semaine - signification $\left.p<0,001\right)(\times 6$ à la $10^{e}$ semaine - signification $\left.p<0,001\right)$; elle représente alors les 63 p. 100 de lactivité du matériel jéjunal des animaux $T$.

Au premier abord, on pourrait relier directement cette élévation d'activité amylasique à la prolifération bactérienne relevée chez ces mêmes animaux (Catala et Bonnafoust, 1974). En effet, nous avons bien observé une multiplication bactérienne mais qui atteint son taux maximum déjà dès le $4^{\mathrm{e}}$ jour postligature ; donc cette prolifération ne peut expliquer, à elle seule, cette variation d'activité. Nous pensons donc, qu'il pourrait apparaitre en plus, des modifications qualitatives de cette flore ef vraisemblablement des processus d'induction au niveau de la bactérie.

En conclusion, nous pouvons constater que la ligature du canal pancréatique entraîne des processus de compensation, relativement précoces ef plus intensément au niveau du matériel intraluminal, par l'intermédiaire des microorganismes, et beaucoup plus tardivement au niveau pariétal. 


\section{Références}

ALPERS D. H., SOLIN M., 1970. Characterization of rat intestinal amylase. Gastroenterology, 586, 833-842.

ALPERS D. H., TEDESCO F. J., 1975. The possible role of pancreatic proteases in the furn-over of intestinal brush border proteins. Biochim. Biophys. Acta, Pays-Bas, 401, 28-40.

ARVANITAKIS C., OLSEN W. A., 1973. Pancreatic diseases and intestinal mucosal enzymes. Gostroenterology, 64 A, 854.

BERNFELD P., 1951. Enzyme of starch degradation and synthesis. Adv. Enzymol., 12, 379.

CATALA J., BONNAFOUS R., 1974. Variations quantitatives de la microflore intestinale du lapin après ligature du canal pancréatique. C. R. Acad. Sci. Paris, sér. D, 278, 3351-3353.

CERDA J. J., PREISER H., CRANE C. K., 1972. Brush border enzymes and malabsorption. Elevated disaccharidases in chronic pancreatic insufficiency with diabete Mellitus. Gastroenterology, 62 A, 841.

MADZAROVOVA-NOHEJLOVA J., 1976. Small bowel disaccharidases activity in chronic pancreatitis. Digestive enzymes. Commun. Est-bilateral Symp., U.S.S.R.-Tchécoslovaquie, 29-31.

Mc MICHAEL H. B., DAHLQUIST A., 1968. Some studies of starch hydrolysis : an interpretation of membrane digestion. Gut, 9, 365-366.

ROBERTS M. C., 1974. Amylase activity in the small intestine of the horse. Rev. veter. Sci., G. B., 17, 400-401. 